\title{
Compensatory mechanisms affect sensorimotor integration during ongoing vocal-motor acts in marmoset monkeys
}

\author{
Thomas Pomberger ${ }^{1,2,3}$, Julia Löschner ${ }^{1,3}$, Steffen R. Hage ${ }^{1, *}$
}

${ }^{1}$ Neurobiology of Vocal Communication, Werner Reichardt Centre for Integrative Neuroscience, University of Tübingen, Otfried-Müller-Str. 25, 72076 Tübingen, Germany

${ }^{2}$ Graduate School of Neural \& Behavioural Sciences - International Max Planck Research

School, University of Tübingen, Österberg-Str. 3, 72074 Tübingen, Germany

${ }^{3}$ These authors contributed equally

*correspondence to steffen.hage@uni-tuebingen.de 


\section{Abstract}

2 In vertebrates, any transmission of vocal signals faces the challenge of acoustic

3 interferences such as heavy rain, wind, animal, or urban sounds. Consequently, several

4 mechanisms and strategies have evolved to optimize the signal-to-noise ratio. Examples to

5 increase detectability are the Lombard effect, an involuntary rise in call amplitude in

6 response to masking ambient noise, which is often associated with several other vocal

7 changes such as call frequency and duration, as well as the animals' capability of limiting

8 calling to periods where noise perturbation is absent. Previous studies revealed rapid vocal

9 flexibility and various audio-vocal integration mechanisms in marmoset monkeys. Using

10 acoustic perturbation triggered by vocal behavior, we investigated whether marmoset

11 monkeys are capable of exhibiting changes in call structure when perturbing noise starts

12 after call onset or whether such effects only occur if noise perturbation starts prior to call

13 onset. We show that marmoset monkeys are capable of rapidly modulating call amplitude

14 and frequency in response to such perturbing noise bursts. Vocalizations swiftly increased

15 call frequency after noise onset indicating a rapid effect of perturbing noise on vocal motor

16 pattern production. Call amplitudes were also affected. Interestingly, however, the

17 marmosets did not exhibit the Lombard effect as previously reported but decreased their call

18 intensity in response to perturbing noise. Our findings indicate that marmosets possess a

19 general avoidance strategy to call in the presences of ambient noise and suggest that these

20 animals are capable of counteracting a previously thought involuntary audio-vocal

21 mechanism, the Lombard effect, presumably via cognitive control processes.

\section{Keywords}

23 Audio-vocal integration; Callithrix jacchus; primate communication; vocal communication; 24 vocal flexibility 


\section{Introduction}

26 Communication between individuals is a crucial aspect for evolutionary success and appears

27 in various forms in nature ranging from olfactory [1,2] to visual [3] to vocal signals [4]. For

28 proper communication, the transmission of a signal sent out by a sender has to be detected

29 and decoded by one or more receivers [5]. Therefore, the sender has to be able to modulate

30 the signal in response to potential masking ambient noise to ensure proper signal

31 transmission. For vocal communication in vertebrates, several mechanisms have evolved to

32 compensate for masking acoustic interferences, such as heavy rain, wind, animal, or urban

33 sounds, leading to changes in temporal and spectral features of the vocal signals [6]. Such

34 vocal modifications can happen involuntarily as well as under volitional control.

35 One of the most efficient mechanisms to increase signal-to-noise ratio in call production is

36 the so-called Lombard effect, i.e., the involuntary increase in call amplitude in response to

37 masking ambient noise [7]. It is often accompanied by a shift in call frequency[8,9] as well as

38 a change in call duration $[10,11]$ and has been shown in many vertebrate species from fish

39 to frogs to birds to mammals including humans [12,13], suggesting that the Lombard effect is

40 an evolutionary old behavior that may have emerged about 450 million years ago. Another

41 successful strategy to increase detectability in a noisy environment is the restraint of call

42 emission to timeslots where noise perturbation is low or absent $[10,14,15]$. This approach

43 renders the modification of call parameters unnecessary and avoids the increased

44 physiological cost of call emission at high intensities that might still be insufficiently

45 increasing signal-to-noise ratio.

46 The common marmoset, a small, highly vocal New World monkey indigenous to the dense

47 rainforests of Brazil, has been shown to exhibit vocal flexibility, such as increasing call

48 intensity $[16,17]$ or increasing the duration of specific calls [17], as well as the attempt to call

49 in silent gaps [14], in the presence of perturbing ambient noise. These findings suggest that

50 while these animals generally seem to prefer avoiding calling in a noisy environment, they do

51 exhibit the involuntary audio-vocal effects discussed above when doing so. This idea is 
52 supported by a recent study showing that marmoset tend to produce single calls instead of

53 call sequences in response to perturbing noise stimuli [18]. Interestingly, marmoset monkeys

54 are also capable of interrupting ongoing vocalizations rapidly after noise perturbation onset

55 [18], overturning decades-old concepts regarding vocal pattern generation [19-21],

56 indicating that vocalizations do not consist of one discrete call pattern but are built of many

57 sequentially uttered units that might be modulated and initiated independently of each other.

58 However, it is yet unclear whether audio-vocal mechanisms, such as the Lombard effect and

59 its accompanied changes in call frequency, can be rapidly elicited in cases where the

60 perturbing noise starts after call onset or whether such effects only occur if noise

61 perturbation starts prior to call onset.

62 In the present study we use acoustic perturbation triggered by the vocal behavior itself to

63 test in a controlled experimental design whether marmosets are capable of rapidly

64 modulating distinct vocal parameters such as call frequency and amplitude in ongoing

65 vocalizations. Performing quantitative measures of resulting adjustments, we show that

66 marmoset monkeys are able to specifically and rapidly modulate call frequency and

67 amplitude as a response to white noise stimuli in ongoing vocal utterances. Hereby, our data

68 indicate that marmosets exhibit a decrease in call amplitude as a result of such noise

69 perturbation, suggesting a mechanism counteracting the rise in amplitude caused by the

70 Lombard effect. 


\section{Results}

72 We measured vocal behavior in marmoset monkeys (Callithrix jacchus, $n=4$ ), a highly vocal

73 New World monkey species, while separated in a soundproofed chamber, with and without

74 acoustic perturbation (Fig. 1A and B). In this setting, marmoset monkeys predominantly

75 produced phee calls (monkey H: 92.0\%, S: 99.1\%, F: 96.8\%, W: 95.6\%), long-distance

76 contact calls, composed of one (so-called single phees), two (double phees), or more phee

77 syllables, to interact with conspecifics [22] (Fig. 1C). Other call types such as trill-phees,

78 twitters, trills, tsik-ekks [22,23], and segmented phees [24] were rarely uttered (all other call

79 types were well below $2.5 \%$ in all monkeys except trill-phees in monkey $\mathrm{H}$ [4.6\%]).

80 We perturbed $2 / 3$ of calls with noise playback after vocal onset to ensure perturbation

81 starting after call initiation (Fig. 1B). To investigate whether perturbation of different

82 frequency bands within the hearing range of the monkeys has different effects on their vocal

83 behavior, we played back five different noise band conditions (broadband noise and

84 bandpass filtered noise bands below [0.1-5 kHz], around [5-10 kHz], or above the

85 fundamental frequency of phee calls [noise bands of $10-15 \mathrm{kHz}$ and $16-21 \mathrm{kHz}$ ] at four

86 different amplitudes [50 dB, $60 \mathrm{~dB}, 70 \mathrm{~dB}, 80 \mathrm{~dB}]$ each). All noise conditions were played

87 back pseudo-randomly in blocks of 30 uttered vocalizations, resulting in 20 calls being

88 perturbed by noise after call onset and 10 calls not being perturbed by noise (control). In

89 total, our monkeys produced 6,298 phees (monkey $F=1544$ phees, $H=1471, S=1631, W$

90 = 1652). Monkeys uttered mostly single and double phees (multi-syllabic phees with more

91 than two syllables were rare or absent: monkey $F=6.5 \%, H=0.4 \%, S=1.3 \%$, absent in $W$ ),

92 with double phee rates between $8.4 \%$ and $55.5 \%$ (mean: $29.5 \% \pm 9.8 \%, \mathrm{n}=4$ monkeys) in

93 the control condition.

94 We first investigated if and how marmosets changed the fundamental frequency of their

95 ongoing phee syllables when perturbed by different noise conditions. We found an increase

96 in first syllable frequencies $(F(3,4904)=6.42, p=2.0 e-04$ for amplitude, $F(4,4904)=20.68$,

$97 \mathrm{p}<0.0001$ for frequency, $\mathrm{n}=3180$ ). Those frequency shifts were significant in the $0.1-5.1 \mathrm{kHz}$ 
98 at $80 \mathrm{~dB}$ noise condition $(38.5 \pm 13.8 \mathrm{~Hz}, \mathrm{p}=1.02 \mathrm{e}-02, \mathrm{n}=168)$, in the two loudest conditions of 99 the $5-10 \mathrm{kHz}$ noise band $(70 \mathrm{~dB}: 56.9 \pm 14.5 \mathrm{~Hz}, \mathrm{p}=3.20 \mathrm{e}-03, \mathrm{n}=165 ; 80 \mathrm{~dB}: 76.7 \pm 16.4 \mathrm{~Hz}$, $100 \mathrm{p}=4.12 \mathrm{e}-08, \mathrm{n}=134$ ), and in all four amplitude conditions of broadband noise (50 dB: $10139.2 \pm 13.7 \mathrm{~Hz}, p=5.03 e-10, n=159 ; 60 \mathrm{~dB}: 68.6 \pm 13.4 \mathrm{~Hz}, p=1.21 \mathrm{e}-08, \mathrm{n}=143 ; 70 \mathrm{~dB}$ : $102104.1 \pm 12.5 \mathrm{~Hz}, \mathrm{p}=3.99 \mathrm{e}-13, \mathrm{n}=135 ; 80 \mathrm{~dB}: 101.7 \pm 16.1 \mathrm{~Hz}, \mathrm{p}=1.66 \mathrm{e}-20, \mathrm{n}=118$; control: $103 \mathrm{n}=1733$; Fig. 2A). The largest frequency shift could be observed for $70 \mathrm{~dB}$ broadband noise, 104 while in the next higher intensity condition $(80 \mathrm{~dB})$, there was no further increase in 105 frequency $(p=1, n=253)$, indicating that marmosets are only capable of altering their 106 fundamental frequency within a certain range. Frequency shifts were not observed in calls 107 that were produced during $10-15 \mathrm{kHz}$ and $16-21 \mathrm{kHz}$ noise band perturbations $(p=1, n=669$ 108 for the $10-15 \mathrm{kHz}$ noise band, $\mathrm{n}=652$ for the $16-21 \mathrm{kHz}$ noise band, Fig. 2A). Second phee 109 syllables showed no significant shift in fundamental frequencies when perturbed by noise $110(F(3,1343)=1.56, p=0.20$ for amplitude, $F(4,1343)=1.24, p=0.29$ for frequency, $n=761$, Fig. 111 2B).

112 Next, we quantified the magnitude of the observed frequency shifts by calculating population 113 effect sizes (ES) of the factors frequency $\left(E_{\text {freq }}\right)$, amplitude $\left(E_{\text {ampl }}\right)$, and the combination of

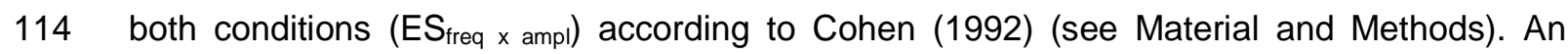
115 effect would be given if the corresponding ES value of a factor was above the threshold of

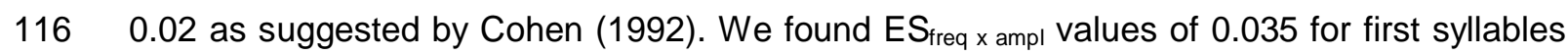
117 and 0.019 for second syllables, indicating an effect for first syllables (Fig. 2A and 2B). ES freq 118 for the first syllable was above the threshold $\left(E_{\text {freq }}=0.023\right)$, while $E S_{a m p l}$ was below $119\left(E_{\mathrm{ampl}}=0.01\right)$, indicating that the shifts in fundamental frequency were mainly correlated with 120 the different noise rather than amplitude conditions.

121 We then tested how fast fundamental frequency shifts occurred within the first phee syllables 122 after noise onset. Therefore, we plotted the mean fundamental frequency courses starting at 123 noise onset times (Fig. 2C and fig. S5). The shortest latency of fundamental frequency 124 shifts within a noise condition was defined as the moment where fundamental frequency 
125 shifts were significant for a minimum of five consecutive milliseconds after noise onset.

126 Shortest latencies were found for the $0.1-5.1 \mathrm{kHz}$ noise condition at $80 \mathrm{~dB}$ (33 ms, $\mathrm{n}=168$ )

127 and all broadband conditions (50 dB: $29 \mathrm{~ms}, \mathrm{n}=159 ; 60 \mathrm{~dB}: 34 \mathrm{~ms}, \mathrm{n}=143 ; 70 \mathrm{~dB}: 25 \mathrm{~ms}$, $128 \mathrm{n}=135 ; 80 \mathrm{~dB}: 25 \mathrm{~ms}, \mathrm{n}=118)$, resulting in a mean latency of $29.2 \pm 1.9 \mathrm{~ms}$.

129 Subsequently, we investigated how call amplitudes changed in response to noise

130 perturbation. We calculated mean amplitude shifts after noise onset for first and second 131 phee syllables (Fig. 3A and fig. 3B). We found a significant decrease in call amplitude for 132 first phee syllables $(F(3,3084)=1.01, p=0.39$ for amplitude, $F(4,3084)=5.3, p=0.0003$ for 133 frequency, $n=2019$ ). These shifts were significant for the two middle intensity levels of the $1340.1-5.1 \mathrm{kHz}$ noise $(60 \mathrm{~dB}:-1.7 \pm 0.5 \mathrm{~dB} p=3.28 \mathrm{e}-03, \mathrm{n}=103 ; 70 \mathrm{~dB}:-2.7 \pm 0.5 \mathrm{~dB}, \mathrm{p}=8.17 \mathrm{e}-04$, $135 \mathrm{n}=119)$ as well as for the two middle intensity levels of the broadband noise $(60 \mathrm{~dB}:-2.3 \pm 0.6$ $136 \mathrm{~dB}, \mathrm{p}=5.15 \mathrm{e}-03, \mathrm{n}=93 ; 70 \mathrm{~dB}:-2.0 \pm 0.6 \mathrm{~dB}, \mathrm{p}=8.59 \mathrm{e}-04, \mathrm{n}=85)$. However, we could not find 137 any systematic increase in amplitude shifts or significant amplitude shifts in any of the five 138 noise conditions ( $n=3093$; Fig. 3A). Furthermore, the combined effect size $\left(E_{\text {freq }} x\right.$ 139 ampl=0.024) was above 0.02 while the effect size for the frequency $\left(E S_{\text {freq }}=0.014\right)$ and 140 amplitude $\left(E S_{a m p l}=0.007\right)$ factors were below 0.02 , indicating that noise perturbation of 141 ongoing first syllables has only a small or no effect on amplitude shifts.

142 However, there was also an amplitude decrease in second phee syllables $(F(3,350)=3.76$, $143 \mathrm{p}=0.011$ for amplitude, $F(4,950)=1.71, p=0.15$ for frequency, $n=554)$. The amplitude shifts in 144 the $0.1-5.1 \mathrm{kHz}$ and $5-10 \mathrm{kHz}$ noise conditions were significant at the highest intensity 145 levels $(-7.2 \pm 1.3 \mathrm{~dB}, \mathrm{p}=3.90 \mathrm{e}-02, \mathrm{n}=19$ and $-7.9 \pm 3.1 \mathrm{~dB}, \mathrm{p}=2.68 \mathrm{e}-03, \mathrm{n}=16$, respectively; fig. 146 3B). Monkeys decreased their call amplitudes in these two conditions with increasing noise 147 intensity levels while no significant call amplitude changes were observed in the other three 148 conditions. All three ES values were above $0.02 \quad\left(E_{\text {freq }} \times\right.$ ampl $=0.064, E S_{\text {freq }}=0.030$, $\left.149 \mathrm{ES}_{\mathrm{ampl}}=0.024\right)$ suggesting an effect of specific noise perturbation on amplitude shifts of 150 second phee syllables in marmoset monkeys. Although it has been already shown that 151 marmoset monkeys show the Lombard effect while producing twitter calls[17], our results 
152 might indicate that marmoset monkeys do not exhibit this reflex when producing phee calls

153 or suppress it and lower their call intensities instead.

154 To test whether our animals are able to show a Lombard effect or suppress it in a noisy 155 environment in general when producing phee calls, we modified our behavioral experiment 156 scheme. We played back all five noise conditions $[0.1-5 \mathrm{kHz}, 5-10 \mathrm{kHz}, 10-15 \mathrm{kHz}, 16-21$

$157 \mathrm{kHz}$, and broadband] at $70 \mathrm{~dB}$ SPL amplitude intensity plus two control conditions with a 158 duration of $180 \mathrm{~s}$ each, resulting in a block of seven pseudorandomized playback conditions 159 with a total duration of $1260 \mathrm{~s}$. In this new experiment our monkeys produced a total of 803 160 phee calls (monkey $F=222$ phees, $H=270, S=158, W=153$ ), which were more commonly 161 uttered $(F=82.5 \%, H=80.4 \%, S=84.0 \%, W=100 \%)$ than other produced call types. The 162 relative amounts of single phees ranged between $34.8 \%$ and $56.3 \%$ and the relative 163 amounts of double phees ranged between $43.71 \%$ and $59.49 \%$. Multi-syllabic phees $(F=$ $1640.5 \%, H=1.9 \%, S=5.7 \%, W=0 \%)$ and segmented phees $(F=0.4 \%, H=2.4 \%, S=0 \%, W$ $165=0 \%$ ) were nearly absent. Monkey $\mathrm{H}$ produced $14.3 \%$ trill-phees and monkeys $\mathrm{F}$ and $\mathrm{S}$ produced $15.2 \%$ and $13.8 \%$ tsik-ekks, respectively. All other call types were below $2.5 \%$ for

167 all monkeys. Under these experimental conditions we found that monkey $\mathrm{W}$ significantly 168 increased its call intensity for both phee syllables when perturbed by noise (first syllable: $1696.4 \pm 0.8 \mathrm{~dB}, \mathrm{p}=1.57 \mathrm{e}-03, \mathrm{n}=107$; second syllable: $8.4 \pm 0.9 \mathrm{~dB}, \mathrm{p}=6.52 \mathrm{e}-03, \mathrm{n}=46$; fig. $3 \mathrm{C}$ and 170 fig. S6), thus, exhibiting the Lombard effect. Furthermore, monkey S significantly decreased 171 the intensity of the second phee syllable and exhibited no changes in call intensity of the first 172 syllable (second syllable: $-4.2 \pm 1.0 \mathrm{~dB}, p=2.15 \mathrm{e}-03, \mathrm{n}=52$; first syllable: $p=0.10, n=68$ ) while 173 monkeys $\mathrm{F}$ and $\mathrm{H}$ showed no significant amplitude change under noise perturbation (first 174 syllable $(H): p=0.89$, second syllable $(H): p=0.15, n=234$; first syllable $(F): p=0.91$, second 175 syllable $(F): p=0.06, n=184)$. Taken together, the present results suggest that marmosets are 176 capable of exhibiting as well as actively suppressing the Lombard effect in a noisy 177 environment during phee call production. 


\section{Discussion}

179 Our results demonstrate that marmoset monkeys show rapid modulation of call parameters 180 in response to perturbing noise bursts presented after call onset. Ongoing phee 181 vocalizations perturbed by ambient noise rapidly increased call frequency in cases where the 182 fundamental frequency was above or directly masked by the perturbing noise. Bandpass183 filtered noise bursts, which did not mask but were above the fundamental frequencies of the 184 calls, had no effect on call frequency. Additionally, call amplitudes of phee calls were 185 affected by low frequency noise bands and broadband noise. Surprisingly, phee calls 186 perturbed after call onset did not exhibit a Lombard effect as previously reported for calls 187 that were produced in constantly presented ambient noise [17,25]. Instead, our monkeys 188 decreased their call intensity in a stepwise function with increasing noise intensity. Our 189 findings suggest a general strategy of avoiding calling in a noisy environment in marmoset 190 monkeys.

191 Effects of ambient noise on call frequency. Noise-dependent shifts in call frequency are 192 not well-studied and relatively poorly understood. Only a few studies have reported a rise in 193 call frequencies with increasing amplitudes of ambient noise in birds and bats $[8,9,26]$ and 194 only one study investigated the effect of different noise bands on call frequencies. In bats, 195 the frequencies of echolocation calls increased significantly for a variety of noise stimuli no 196 matter whether they were directly masking the call's fundamental frequency or presented 197 below the dominant call frequency [8]. In contrast, the present study shows that in 198 marmosets, call frequency was predominantly only affected when we directly masked the 199 calls fundamental frequency. As a result, the strongest rise in call frequencies were found for 200 high noise amplitudes. These findings suggest that the observed rises in call frequencies are 201 an audio-vocal mechanism elicited to increase call detectability in a noisy environment, as 202 has been found in previous studies involving birds [27-29]. Here, it has been predicted that 203 shifts in song frequencies of about $200 \mathrm{~Hz}$ increase call detectability by about 10 to $20 \%$ 204 [28], which is mainly due to the fact that the spectrum of environmental noise generally 
205 shows a decay in amplitude with increasing frequency [28-31]. In the present study, shifts in 206 call frequency occurred with a mean latency of about $30 \mathrm{~ms}$ after noise onset suggesting a 207 rapid underlying neural mechanism for frequency modulation. Such fast responses to 208 ambient noise have yet only been found in echolocating bats, which exhibit an increase in 209 call amplitude in about $30 \mathrm{~ms}$ after noise onset as well [32].

210 Effects of ambient noise on call amplitude. Despite the positive effect of rises in call 211 frequency on signal detectability, the most effective mechanism to improve signal to noise 212 ratio in a noisy environment during vocal production is the Lombard effect, i.e., the 213 involuntary rise in call amplitude as a response to masking noise $[12,13]$. In the present 214 study, noise perturbation starting after phee call onset had no systematic effect on call 215 amplitude of the first syllable, i.e., the syllable during which noise perturbation started. In 216 cases in which significant shifts occurred, call amplitude did not increase, as expected, but 217 decreased with small effect sizes. This effect was stronger for the second syllables of the 218 phee calls, in which a strong decrease in call amplitude could be observed for low frequency 219 noise conditions. Consequently, call intensity decreased in a stepwise function with 220 increasing noise intensity suggesting a direct effect of noise intensity on call amplitude. In 221 contrast to our study, the Lombard effect has been observed in marmoset monkeys in a 222 previous study [17]. This apparent discrepancy might be explained by the different call types 223 that were investigated in both studies. While we focused on phee calls, a high amplitude call 224 that is produced at the upper end of the amplitude scale [16], the earlier study investigated 225 the twitter call, a vocalization that is produced at lower amplitude intensities [17].

226 Our results suggest an audio-vocal integration mechanism in marmoset monkeys that is 227 capable of counteracting the Lombard effect. Such a mechanism has been already shown to 228 exist in vocal production learners such as birds and humans [33-36] and seems to be mainly 229 driven by higher-order cognitive processes including cortical structures [13].

230 Vocal flexibility during perturbing noise in marmoset monkeys. Current studies have 231 revealed a high degree of vocal flexibility in marmoset monkeys [37], allowing them to 
232 control when[14], where[38], and what to vocalize[39]. In addition, recent studies revealed

233 that marmosets are able to modulate distinct call parameters in response to acoustic 234 feedback $[18,40]$. This vocal flexibility allows marmosets to avoid calling in the presence of 235 environmental noise and predominantly initiate their vocalizations in silent periods [14]. In a 236 previous study, we demonstrated that marmosets interrupt their vocalizations shortly after 237 noise onset when perturbation starts after vocal onset [18], supporting the idea that these 238 animals tend to avoid calling in ambient noise. Such call interruptions, however, were rare 239 (2.6\% of all calls), indicating stark neuronal and/or anatomical constraints in exhibiting such 240 behavior [18] and resulting in a large fraction of phee calls being perturbed by noise bursts. 241 In the present study, we show that the call amplitude of such vocalizations are lower.

242 We suggest that marmoset monkeys exhibit this vocal behavior in a noisy environment to 243 reduce the physiological costs of high intensity phee calls. Marmoset phee calls are elicited 244 at high intensities above $100 \mathrm{~dB}$ SPL, resulting in high muscle tensions encompassing 245 almost the entire animal's body during call production (own observation). Therefore, 246 mechanisms might have evolved in these animals that ensure the proper transmission of 247 these high energetic calls resulting in calling in silent gaps and decreasing call intensity in 248 situations in which sufficient detectability might be potentially diminished, such as during the 249 presence of ambient noise.

Mechanisms counteracting involuntary audio-vocal effects need cognitive control.

251 Based on the current work and earlier studies [14,18], we propose a hypothetical neuronal 252 model suggesting various audio-vocal control mechanism involving cortical, subcortical, and 253 corticofugal connections capable of modulating vocal behavior in a noisy environment (Fig. 254 4). In accordance to earlier work [41,42], our model consists of a volitional articulatory motor 255 network originating in the prefrontal cortex (PFC) cognitively controlling vocal output of a 256 phylogenetically conserved primary vocal motor network predominantly consisting of a 257 subcortical neuronal network. The vocal motor network can be modulated by auditory 258 structures on several cortical and subcortical brain levels [13]. The decision to initiate or 
259 suppress a call, as well as counteracting an involuntary effect (Lombard effect), needs

260 cognitive control. The ability to interrupt calls or modulate call parameters as a response to

261 perturbing noise might be controlled by both subcortical mechanisms and corticofugal

262 projections. Neurophysiological studies will now have to elucidate at which brain levels

263 audio-vocal integration mechanisms exist that explain the observed capabilities of marmoset

264 monkeys to counteract a previously thought involuntary audio-vocal mechanism, the 265 Lombard effect. 


\section{Material and Methods}

267 Animal Housing and Maintenance. Four adult marmoset monkeys (Callithrix jacchus) were

268 used in the present study. Monkeys were usually kept in different sex pairs and were all born

269 in captivity. The animals had ad libitum access to water and were fed on a restricted food

270 protocol including a daily basis of commercial pellets, fruits, vegetables, mealworms, and

271 locusts. Additional treats, such as marshmallows or grapes, were used as positive

272 reinforcements to transfer the animals from their home cage to the experimental cage.

273 Environmental conditions in the animal husbandry were maintained at a temperature of

$27426^{\circ} \mathrm{C}, 40-60 \%$ relative humidity, and a $12 \mathrm{~h}: 12 \mathrm{~h}$ day/night cycle. All animal handling

275 procedures were in accordance with the guidelines for animal experimentation and

276 authorized by the national authority, the Regierungspräsidium Tübingen. All vocalizations

277 analyzed in this study are a fraction of calls that have been recorded in a previous study

278 (Pomberger et al. 2018).

279 Experimental Setup and Procedure. The vocal behavior of four animals was recorded in a 280 soundproof chamber in response to noise playback that was initiated after vocal onset as reported earlier (Pomberger et al., 2018). Briefly, the animals were transferred into a recording cage $(0.6 \times 0.6 \times 0.8 \mathrm{~m})$, which was placed in a soundproofed chamber, with ad

283 libitum access to water and food pellets throughout the recording period. In this behavioral 284 setup, marmoset monkeys predominantly produce phee calls to interact with conspecifics 285 (phee ratio within all uttered calls; monkey S: $99.1 \%, \mathrm{H}: 92.0 \%$, W: $95.6 \%$, F: $96.8 \%$ ). 286 Other call types such as trill-phees, twitter, trills, tsik-ekks [22], or segmented phees [24] 287 were only rarely uttered (ratios were well below $2.5 \%$ for all other call types in all monkeys 288 except trill-phees in monkey H [4.6\%]). Monkeys produced a mean of $118 \pm 9$ (monkey S), $289167 \pm 31(H), 117 \pm 10(W)$, and $87 \pm 7(F)$ phee calls per session. The vocal behavior of each 290 individual monkey was recorded once a day in sessions ranging between one and two hours 291 in duration. Data were collected in sessions at various times during the day between 11 am 292 and 5 pm. Recordings were performed for 10-28 days (mean: 17 \pm 3 days) for each individual 
293 animal. The monkey's behavior was constantly monitored and observed with a video camera

294 (ace acA1300-60gc, Basler, Germany with 4.5-12.5 mm CS-Mount Objective H3Z4512CS-

295 IR 1/2, Computar, Japan) placed on top of the cage and recorded with standard software

296 (Ethovision XT version 4.2.22, Noldus, the Netherlands). The vocal behavior was collected

297 with eight microphones (MKH 8020 microphone with MZX 8000 preamplifier, Sennheiser,

298 Germany), which were positioned in an octagonal design around the cage (Fig. 1A),

299 digitized using an A/D interface (Octacapture, Roland, Japan; sample rate: $96 \mathrm{kHz}$ ), and

300 recorded using standard software (Avisoft-Recorder, Avisoft Bioacoustics, Germany). A

301 custom-written program (OpenEX, Tucker-Davis Technologies, U.S.A.) running on a

302 workstation (WS-X in combination with an RZ6D multi I/O processor, Tucker-Davis

303 Technologies, U.S.A.) monitored the vocal behavior in real-time via an additional

304 microphone (MKH 8020 microphone with MZX 8000 preamplifier, Sennheiser, Germany)

305 placed on top of the cage, which automatically detected vocalizations through online

306 calculation of several acoustic parameters, such as call intensity, minimum duration of call

307 intensity duration, call frequency, and several spectral features. The median vocal detection

308 rate was well above $99 \%$ and three out of four vocalizations were detected within the first

309146 ms after call onset (Fig. 1B).

310 The eight microphones positioned around the cage were installed to ensure precise

311 calculation of $\mathrm{dB}$ SPL values of vocalizations with a corresponding microphone being

312 positioned in front of the monkey (for detail see below).

313 For two out of three uttered vocalizations, we played back noise bursts of different

314 frequency-bands and amplitudes via a loudspeaker (MF1 Multi-Field Magnetic Speakers,

315 Tucker-Davis Technologies, U.S.A.) positioned on top of the cage, immediately after vocal

316 detection. Noise bursts had a duration of $4 \mathrm{~s}$ (including $10 \mathrm{~ms}$ rise times) to ensure noise

317 perturbation throughout the first phee syllable as well as the initiation of the second syllable

318 (Fig. 1C). Five different noise band conditions (broadband noise and bandpass filtered noise

319 bands: $0.1-5.1 \mathrm{kHz}, 5-10 \mathrm{kHz}, 10-15 \mathrm{kHz}$, and $16-21 \mathrm{kHz}$ ) were played back at four 
320 different amplitudes $(50 \mathrm{~dB}, 60 \mathrm{~dB}, 70 \mathrm{~dB}, 80 \mathrm{~dB})$ each. All 20 noise conditions were played

321 back pseudo-randomly in blocks of 30 uttered vocalizations, resulting in 20 calls being

322 perturbed with noise after call onset and 10 calls without noise playback remaining 323 unaffected (control). After one block ended, a new block was generated. Noise playback

324 generation and presentation were performed with the same custom-written software used for 325 call detection.

326 Data Analysis. We programmed a custom-written GUI (Matlab, Mathworks, U.S.A.) to clock 327 Avisoft, Noldus, and TDT recordings offline and to extract the detected calls from the 328 recording channel with the best SNR. Vocal onset to offset were manually flagged as well as 329 noise onset times using the aligned sono- and spectrogram of vocalizations. We used a 330 Hanning window with a 512-window size, 1024 FFT, overlap of $25 \%$, and temporal resolution 331 of one millisecond. We only considered first phee syllables for calculation that were 332 detected/perturbed within $200 \mathrm{~ms}$ of call onset and with a minimum duration of $800 \mathrm{~ms}$. 333 Consequently, first phee syllables that were interrupted directly after noise onset as 334 previously reported in an earlier study [18] were excluded from further analysis. Second 335 phee syllables were only analyzed if they had a minimum duration of $500 \mathrm{~ms}$. In rare cases, 336 call termination could not be visually detected due to overlapping noise (mostly during the 80 $337 \mathrm{~dB}$ SPL condition). These calls were also excluded from further analysis.

338 After labelling a call, peak frequencies of the fundamental component were automatically 339 calculated within one-millisecond time bins (8192 FFT, $96 \mathrm{kHz}$ sample rate resulting in a 340 frequency resolution of $11.71 \mathrm{~Hz}$ ). In cases where the SNR between the call amplitude and 341 playback noise was not high enough for automatic fundamental peak frequency calculation, 342 frequency trajectories were calculated by manually setting call frequencies at several time 343 points and interpolating call frequencies in between the set values. The accuracy of manual 344 labelling compared to automatic calculation of peak frequencies was high and median 345 differences between both techniques below the frequency resolution used (Fig. S1). 
346 Call amplitudes were calculated for all phee calls during which the animals did not move

347 their heads during call production. For these calls, head positions were manually labelled by

348 marking the two white ear tufts in the GUI (see Fig. 1B). Next, a perpendicular line starting

349 at the center of the later connection was used to compute angles of the microphones

350 indicating the monkey's relative head position. The microphone with the smallest angle to

351 the perpendicular line was used for further calculation (Fig. 1B). Calls that were uttered in

352 the rare cases where the angle between the front of the monkey's head and the microphone

353 was more than 45 degrees were excluded from further analysis. Furthermore, phee calls that

354 were uttered during head movements of the animal were not used for amplitude calculations

355 and only considered for fundamental frequency calculation resulting in a larger data set for

356 frequency analysis.

357 From the recordings of the microphone foremost in front of the animal, call amplitude 358 trajectories (in $\mathrm{dB} S \mathrm{SPL}$ ) were calculated using a sliding window approach (window size: 25 $359 \mathrm{~ms}$; step length: $1 \mathrm{~ms}$ ). Sound levels of the recorded playback noise were determined for all 360 conditions and subtracted from the call amplitude measurements taken, using a modification 361 of the spectral noise subtraction method [43]. Briefly, we first calculated an estimate for each 362 noise band by calculating the mean of ten recordings of one noise condition for each 363 microphone. Then, we subtracted this noise estimate in the spectral dimension from noise 364 perturbed parts of a call (i.e., from noise onset to the end of the call) and corrected the 365 outcome as shown in formula (1), where $P_{S}(w)$ is the spectrogram of the signal and the 366 noise, $P_{n}(w)$ the spectrogram of the noise estimate and $P_{S}^{\prime}(w)$ the modified signal spectrum.

367 Alpha is defined as the subtraction factor and beta as the spectral floor parameter.

$$
\begin{aligned}
& D(w)=P_{S}(w)-\alpha P_{n}(w) \\
& P_{s}^{\prime}(w)=\left\{\begin{array}{cl}
D(w), & \text { if } D(w)>\beta P_{n}(w) \\
\beta P_{n}(w), & \text { otherwise } \\
\alpha \leq 1, & 0<\beta \ll 1
\end{array}\right.
\end{aligned}
$$

369 Alpha and beta were calculated using the following equation: 


$$
\begin{aligned}
& \alpha=\alpha_{0}-\frac{S N R}{S} \\
& 5 \leq S N R \leq 20
\end{aligned}
$$

371 According to Berouti et al. [43], we chose alpha $a_{0}=4$ and $s=20 / 3$ as a best fit for proper

372 amplitude calculation. A simple empirical test verified the method; a control phee was played 373 and recorded in the recording chamber ten times with broadband noise $70 \mathrm{~dB}$ SPL, ten times 374 with a $5-10 \mathrm{kHz}$ noise band and ten times under control conditions (no noise). As reported 375 previously, differences between conditions of $<1 \mathrm{~dB}$ can be assumed to be negligible [44]. In 376 our case, median differences between control and both noise conditions were below $1 \mathrm{~dB}$ 377 (broadband: $0.8 \mathrm{~dB}, 5-10 \mathrm{kHz}$ noise band: $0.3 \mathrm{~dB}$; Figs. S2A and B) indicating successful 378 performance of the used method. The distance of the animal's head to the microphone was considered by adding a distance factor directly after noise subtraction to the measurements

380 resulting in a standardized amplitude trajectory (in $\mathrm{dB}$ ) of each call as produced $10 \mathrm{~cm}$ in 381 front of the animal's head.

382 Frequency/amplitude calculation and normalization. Mean fundamental frequency 383 values were obtained with a sliding window approach (window size: $10 \mathrm{~ms}$, step size: $1 \mathrm{~ms}$ ). 384 We then calculated the mean of the fundamental frequency in a 20-5 ms time window prior 385 to noise onset (for the noise conditions) and call detection (for the control condition) for each 386 individual call and subtracted this value from each of the frequency values after noise onset.

387 Finally, all values of calls in the noise conditions were normalized by subtracting the mean of 388 the respective frequency value of the control condition. Amplitude values were calculated in 389 a similar way. Here, we also calculated the mean amplitude for each individual call in a 20-5 390 ms time window prior to noise onset and subtracted these values from the mean amplitude 391 values after noise onset. According to the frequency normalization, we then normalized all 392 amplitude values by subtracting the mean of the amplitude values from the corresponding 393 values in the control condition. For the $180 \mathrm{~s}$ noise experiment, we used the calculated 394 amplitude values as described above in data analysis. 
395 Phee call discrimination models. Marmoset monkeys tend to interrupt their phee calls 396 after the first syllable in response to noise perturbation [18]. For perturbed phee calls, we 397 consequently assumed that a substantial number of single phees had to be interrupted double phees. Recently, it has been shown that single phees and the first syllables of double phees significantly differ in a number of call parameters, such as call frequency and duration

400 [20]. We therefore had to find a way to distinguish single phee calls that were interrupted 401 double phees from original single phees prior to data normalization. To address this, we 402 used the findings of Miller and colleagues [20] that suggested that early peak frequencies 403 and durations of phee calls are sufficient to predict whether a phee call consists out of one or 404 two syllables. Additionally, we found that this is also true for early amplitude values of a call. 405 We applied a quadratic classification model (MATLAB) to discriminate between single and 406 double phees with a two-dimensional classifier for fundamental frequency analysis using 1st 407 syllable durations and peak frequencies at $25 \mathrm{~ms}$ after call onset for frequency analyses 408 (Fig. S3). Since we observed that early amplitude values are also a good predictor (Fig. S4), 409 we used a three-dimensional classifier with call amplitude values at $25 \mathrm{~ms}$ after call onset as 410 the third measure for amplitude analyses (Fig. S4). Basically, in a first step the mean, $\mu_{\mathrm{k}}$, 411 and covariance matrix, $\Sigma_{\mathrm{k}}$, of each class is calculated from all control values to obtain the 412 density function of the multivariate normal at a point, $x$, using the following formula:

413

$$
P(x \mid k)=\frac{1}{\left(2 \pi\left|\Sigma_{k}\right|\right)^{1 / 2}} \exp \left(-\frac{1}{2}\left(x-\mu_{k}\right)^{T} \sum_{k}^{-1}\left(x-\mu_{k}\right)\right)
$$

414 Where $\left|\Sigma_{k}\right|$ is the determinant of $\Sigma_{k}$, and $\Sigma_{k}^{-1}$ is the inverse matrix. Using the prior probability $415 P(k)$ of class $k$ and $P(x)$ as a normalization constant we obtain the posterior probability $416 \hat{P}(k \mid x)$ that a point $x$ belongs to class $k$ based on:

$$
\hat{P}(k \mid x)=\frac{P(x \mid k) P(k)}{P(x)}
$$


418 These results are then used to classify our phee calls into single and double phees by

419 minimizing the expected classification cost using:

420

$$
\hat{y}=\arg \min _{y=1, \ldots, K} \sum_{k=1}^{K} \hat{P}(k \mid x) C(y \mid k)
$$

421 Where $\hat{y}$ is the predicted classification, $K$ is the number of classes, and $C(y \mid k)$ is the cost of 422 classifying an observation as $y$ when its true class is $k$. In total, the loss for the 2D 423 classification was between $10.8 \%$ and $23.2 \%$ (mean: $15.1 \pm 2.8$ ) and for the 3D classification 424 between $6.8 \%$ and $15.7 \%$ (mean: $12.3 \pm 1.9$ ) for each monkey.

425 Statistical analysis. Statistical analyses were performed with MATLAB (2016b, MathWorks, 426 Natick, MA). We performed a two-way ANOVA to test for significant differences in shifts of 427 fundamental call frequency and amplitude within all noise band conditions (alpha $=0.05$, 428 Bonferroni corrected). Effect sizes (ES) were calculated using the following formula:

$$
f_{p}^{2}=\frac{\eta_{p}^{2}}{1-\eta_{p}^{2}}
$$

429

430 Where $f_{p}^{2}$ represents the effect size of factor $\boldsymbol{p}$ and $\eta_{p}^{2}$ is calculated as:

431

$$
\frac{\text { explained sum of squares of } \boldsymbol{p}}{\text { (explained sum of squares of } \boldsymbol{p}+\text { residual sum of squares })}
$$

432 Data availability. All data needed to evaluate the conclusions in the paper are present in the 433 paper. Additional data related to this paper may be requested from the corresponding 434 author.

435 


\section{Author Contributions}

S.R.H. conceived the study; T.P. and S.R.H designed the experiments; T.P. and J.L. conducted the experiments and performed data analyses; all authors interpreted the data and wrote the manuscript. S.R.H. provided the animals and supervised the project.

\section{Acknowledgments}

We thank John Holmes for proofreading. This work was supported by the Werner Reichardt Centre for Integrative Neuroscience $(\mathrm{CIN})$ at the Eberhard Karls University of Tübingen (CIN is an Excellence Cluster funded by the Deutsche Forschungsgemeinschaft within the framework of the Excellence Initiative EXC 307). 


\section{References}

1. Poldrack RA, Farah MJ. 2015 Progress and challenges in probing the human brain. Nature 526, 371-379.

2. Stockhorst U, Pietrowsky R. 2004 Olfactory perception, communication, and the noseto-brain pathway. Physiol. Behav. 83, 3-11.

3. Osorio D, Vorobyev M. 2008 A review of the evolution of animal colour vision and visual communication signals. Vision Res. 48, 2042-2051.)

4. Ackermann H, Hage SR, Ziegler W. 2014 Brain mechanisms of acoustic communication in humans and nonhuman primates: An evolutionary perspective. Behav. Brain Sci. 37, 529-546.

5. Bradbury JW, Vehrencamp SL. 1998 Principles of Animal Communication.

6. Brumm H, Slabbekoorn H. 2005 Acoustic Communication in Noise. Adv. Study Behav. 35, 151-209.

7. Lombard E. 1911 Le signe de l'elevation de la voix. Ann. Mal. L'Oreille du Larynx XXXVII, 101-119.

8. Hage SR, Jiang T, Berquist SW, Feng J, Metzner W. 2013 Ambient noise induces independent shifts in call frequency and amplitude within the Lombard effect in echolocating bats. Proc. Natl. Acad. Sci. U. S. A. 110, 4063-4068.

9. Osmanski MS, Dooling RJ. 2009 The effect of altered auditory feedback on control of vocal production in budgerigars ( Melopsittacus undulatus ). J. Acoust. Soc. Am. 126, 911-919.

10. Brumm H. 2006 Signalling through acoustic windows: Nightingales avoid interspecific competition by short-term adjustment of song timing. J. Comp. Physiol. A Neuroethol. Sensory, Neural, Behav. Physiol. 192, 1279-1285.

11. Luo J, Goerlitz HR, Brumm H, Wiegrebe L. 2015 Linking the sender to the receiver: Vocal adjustments by bats to maintain signal detection in noise. Sci. Rep. 5, 1-11.

12. Brumm H, Zollinger A. 2011 The evolution of the Lombard effect: 100 years of psychoacoustic research. Behaviour 148, 1173-1198.

13. Luo J, Hage SR, Moss CF. 2018 The Lombard Effect: From Acoustics to Neural Mechanisms. Trends Neurosci. 41, 938-949.

14. Roy S, Miller CT, Gottsch D, Wang X. 2011 Vocal control by the common marmoset in the presence of interfering noise. J. Exp. Biol. 214, 3619-3629.

15. Zelick RD, Narins PM. 1982 Analysis of acoustically evoked call suppression behaviour in a neotropical treefrog. Anim. Behav. 30, 728-733.

16. Eliades SJ, Wang X. 2012 Neural correlates of the lombard effect in primate auditory cortex. J. Neurosci. 32, 10737-48. 
17. Brumm H. 2004 Acoustic communication in noise: regulation of call characteristics in a New World monkey. J. Exp. Biol. 207, 443-448.

18. Pomberger T, Risueno-Segovia C, Löschner J, Hage SR. 2018 Precise Motor Control Enables Rapid Flexibility in Vocal Behavior of Marmoset Monkeys. Curr. Biol. 28, 788794.e3.

19. Miller CT, Flusberg S, Hauser MD. 2003 Interruptibility of long call production in tamarins: implications for vocal control. J. Exp. Biol. 206, 2629-2639.

20. Miller CT, Eliades SJ, Wang X. 2009 Motor planning for vocal production in common marmosets. Anim. Behav. 78, 1195-1203.

21. Egnor SER, Iguina CG, Hauser MD. 2006 Perturbation of auditory feedback causes systematic perturbation in vocal structure in adult cotton-top tamarins. J. Exp. Biol. 209, 3652-3663.

22. Agamaite JA, Chang C-J, Osmanski MS, Wang X. 2015 A quantitative acoustic analysis of the vocal repertoire of the common marmoset (Callithrix jacchus). $J$. Acoust. Soc. Am. 138, 2906-2928.

23. Pistorio AL, Vintch B, Wang X. 2006 Acoustic analysis of vocal development in a New World primate, the common marmoset ( Callithrix jacchus ). J. Acoust. Soc. Am. 120, 1655-1670.

24. Zürcher Y, Burkart JM. 2017 Evidence for Dialects in Three Captive Populations of Common Marmosets (Callithrix jacchus). Int. J. Primatol. 38, 780-793.

25. Egnor SR, Hauser M. 2006 Noise-induced vocal modulation in cotton-top tamarins (Saguinus oedipus). Am. J. Primatol. 68, 1183-1190.

26. Schuster S, Zollinger SA, Lesku JA, Brumm H. 2012 On the evolution of noisedependent vocal plasticity in birds. Biol. Lett. 8, 913-916.

27. Bermúdez-Cuamatzin E, Ríos-Chelén AA, Gil D, Garcia CM. 2011 Experimental evidence for real-time song frequency shift in response to urban noise in a passerine bird. Biol. Lett. 7, 36-38.

28. Nemeth E, Brumm H. 2010 Birds and Anthropogenic Noise: Are Urban Songs Adaptive? Am. Nat. 176, 465-475.

29. Pohl NU, Leadbeater E, Slabbekoorn H, Klump GM, Langemann U. 2012 Great tits in urban noise benefit from high frequencies in song detection and discrimination. Anim. Behav. 83, 711-721.

30. Halfwerk W, Slabbekoorn H. 2009 A behavioural mechanism explaining noisedependent frequency use in urban birdsong. Anim. Behav. 78, 1301-1307.

31. Pohl NU, Slabbekoorn H, Klump GM, Langemann U. 2009 Effects of signal features and environmental noise on signal detection in the great tit, Parus major. Anim.

Behav. 78, 1293-1300. 
32. Luo J, Kothari NB, Moss CF. 2017 Sensorimotor integration on a rapid time scale. Proc. Natl. Acad. Sci. 114, 6605-6610.

33. Kobayasi KI, Okanoya K. 2003 Context-dependent song amplitude control in Bengalese finches. Neuroreport 14, 521-524.

34. Pick HL, Siegel GM, Fox PW, Garber SR, Kearney JK. 1989 Inhibiting the Lombard effect. J. Acoust. Soc. Am. 85, 894-900.

35. Therrien AS, Lyons J, Balasubramaniam R. 2012 Sensory Attenuation of SelfProduced Feedback: The Lombard Effect Revisited. PLoS One 7, e49370.

36. Vinney LA, van Mersbergen M, Connor NP, Turkstra LS. 2016 Vocal Control: Is It Susceptible to the Negative Effects of Self-Regulatory Depletion? J. Voice 30, 638.e21-638.e31.

37. Ghazanfar AA, Liao DA, Takahashi DY. 2019 Volition and learning in primate vocal behaviour. Anim. Behav. , 1-9.

38. Choi JY, Takahashi DY, Ghazanfar AA. 2015 Cooperative vocal control in marmoset monkeys via vocal feedback. J. Neurophysiol. 114, 274-283.

39. Liao DA, Zhang YS, Cai LX, Ghazanfar AA. 2018 Internal states and extrinsic factors both determine monkey vocal production. Proc. Natl. Acad. Sci. 115, 201722426.

40. Eliades SJ, Tsunada J. 2018 Auditory cortical activity drives feedback-dependent vocal control in marmosets. Nat. Commun. 9, 2540.

41. Hage SR, Nieder A. 2016 Dual Neural Network Model for the Evolution of Speech and Language. Trends Neurosci. 39, 813-829.

42. Hage SR. 2019 Precise vocal timing needs cortical control. Science. 363, 926-928.

43. Berouti M, Schwartz R, Makhoul J. 1978 Enhancement of speech corrupted by acoustic noise. In ICASSP '79. IEEE International Conference on Acoustics, Speech, and Signal Processing, pp. 208-211.

44. Brumm H, Schmidt R, Schrader L. 2009 Noise-dependent vocal plasticity in domestic fowl. Anim. Behav. 78, 741-746. 


\section{Figures}

A

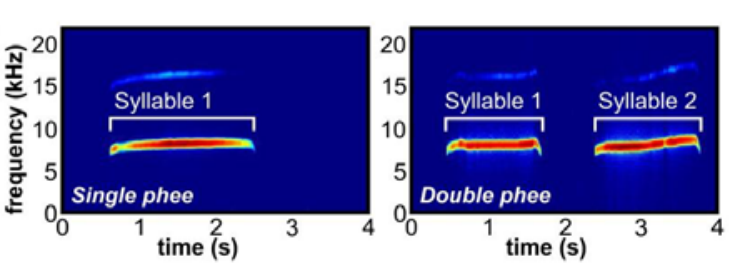

B

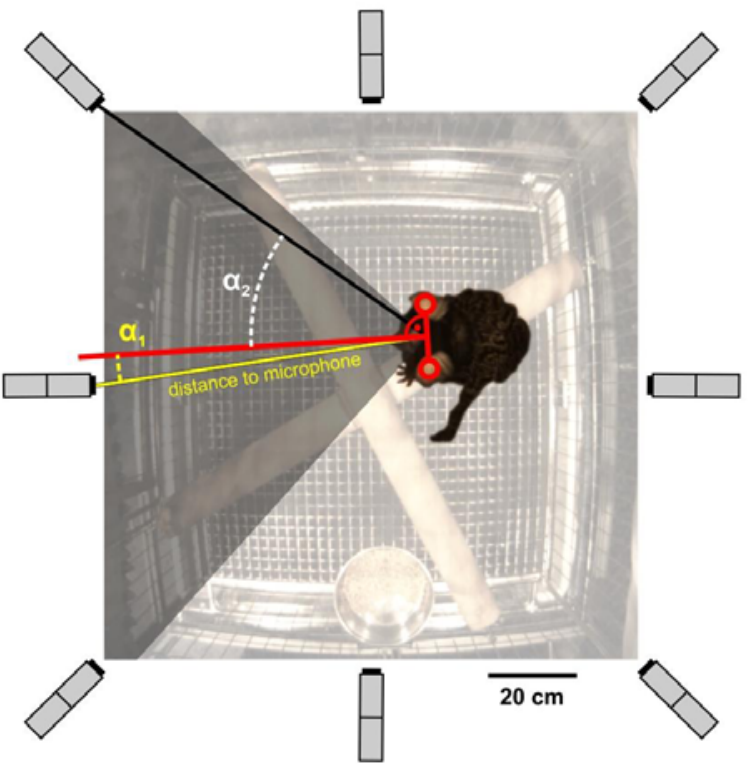

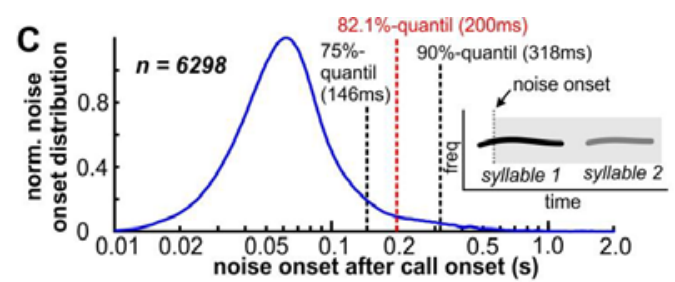

D

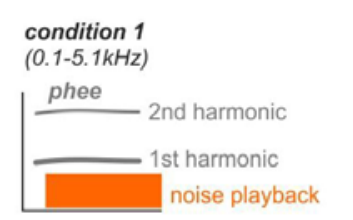

condition 2 $(5-10 \mathrm{kHz})$

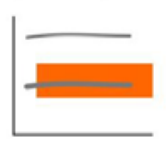

condition 3

condition 4 $(10-15 \mathrm{kHz}) \quad(16-21 \mathrm{kHz})$

condition 5
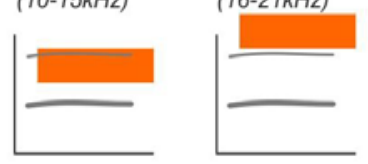

(broadband)

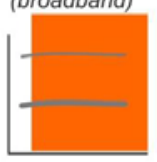

E
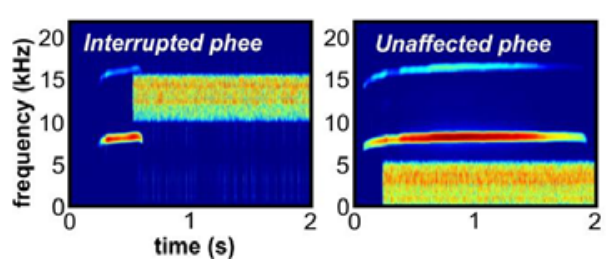

Figure 1: Experimental setup and design. (A) Exemplary spectrograms of single and double phee calls. (B) The vocal behavior of monkeys was recorded in a soundproof chamber. The behavior was continuously monitored and recorded. The red line shows the monkey's head position in relation to the two closest microphones (yellow and black line). The acoustic signal recorded with the microphone closest to being directly in front of the monkey's head (i.e., the smallest angle between the monkey's perpendicular and the microphone) was used for amplitude calculation. (C) Relative vocal detection distribution over time (s). (D) Noise condition overview with masking properties. (E) Exemplary spectrograms of an interrupted single phee (10-15 kHz noise condition) and unaffected phee $(0.1-5 \mathrm{kHz}$ noise condition). 

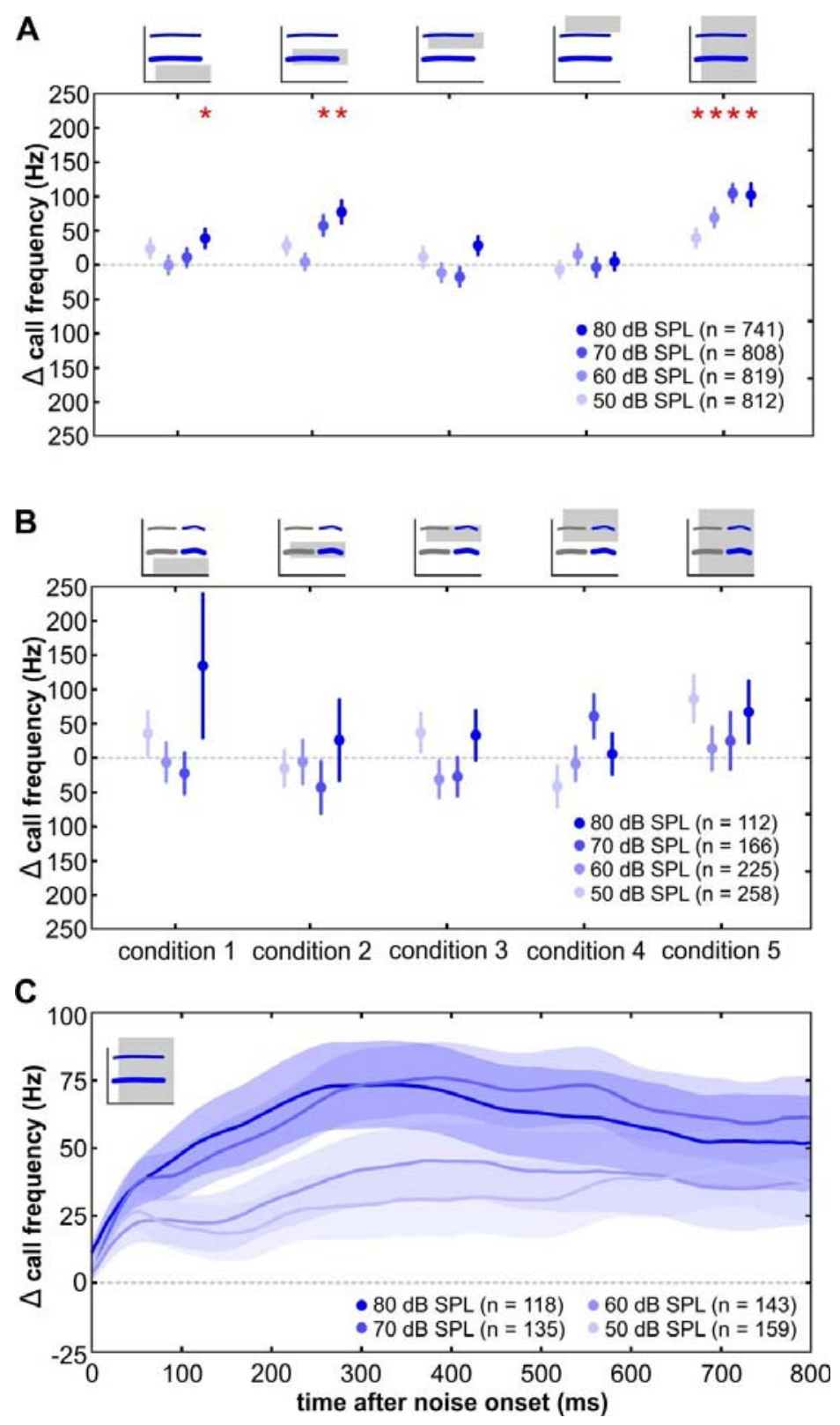

Figure 2: Increasing frequency shifts in response to noise bursts. $\Delta$ call frequency $(\mathrm{Hz})$ per corresponding noise condition normalized to control data (dashed lines). Mean of median frequencies after noise onset of each call pooled over all monkeys \pm SEM $(\mathbf{A})$ for first phee syllables 0-800 ms after noise onset (B) for second phee syllables 100-400 ms after second syllable onset. (C): First syllables mean $\Delta$ call frequency courses $(\mathrm{Hz}) \pm \mathrm{SEM}$ of all amplitude conditions during broadband noise over time after noise onset (ms). Asterisks denote significant differences. 

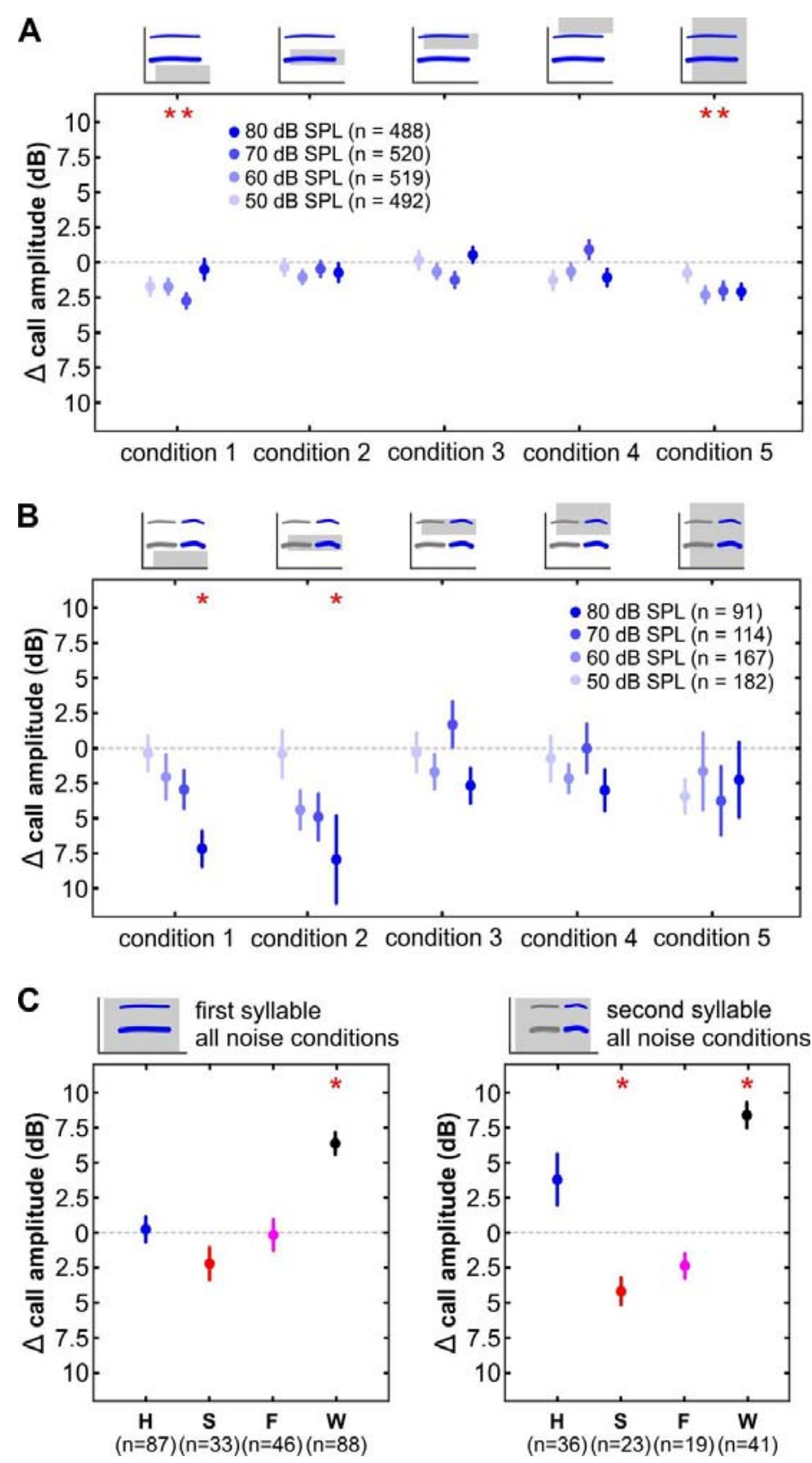

Figure 3: Decreasing amplitude shifts in response to noise bursts. $\Delta$ call amplitude (dB) per corresponding noise condition normalized to control data (dashed lines). Mean of median amplitudes after noise onset of each call pooled over all monkeys \pm SEM (A) for first phee syllables and (B) for second phee syllables. Over all noise conditions pooled max $\Delta$ amplitudes $(\mathrm{dB}) \pm$ SEM during $180 \mathrm{~s}$ noise per monkey (C) for first syllables and (D) for second syllables. Asterisks denote significant differences. 
(1) no call

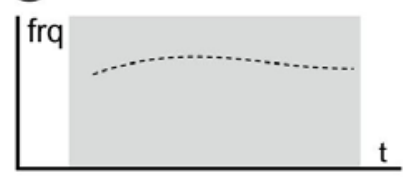

(2) call interruption

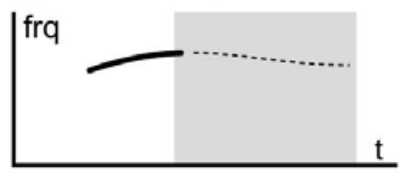

(3) frequency shift

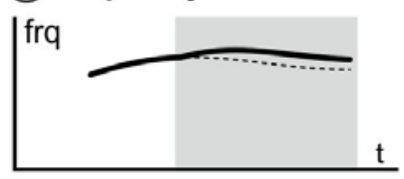

(4) amplitude modulation (4) second syllable
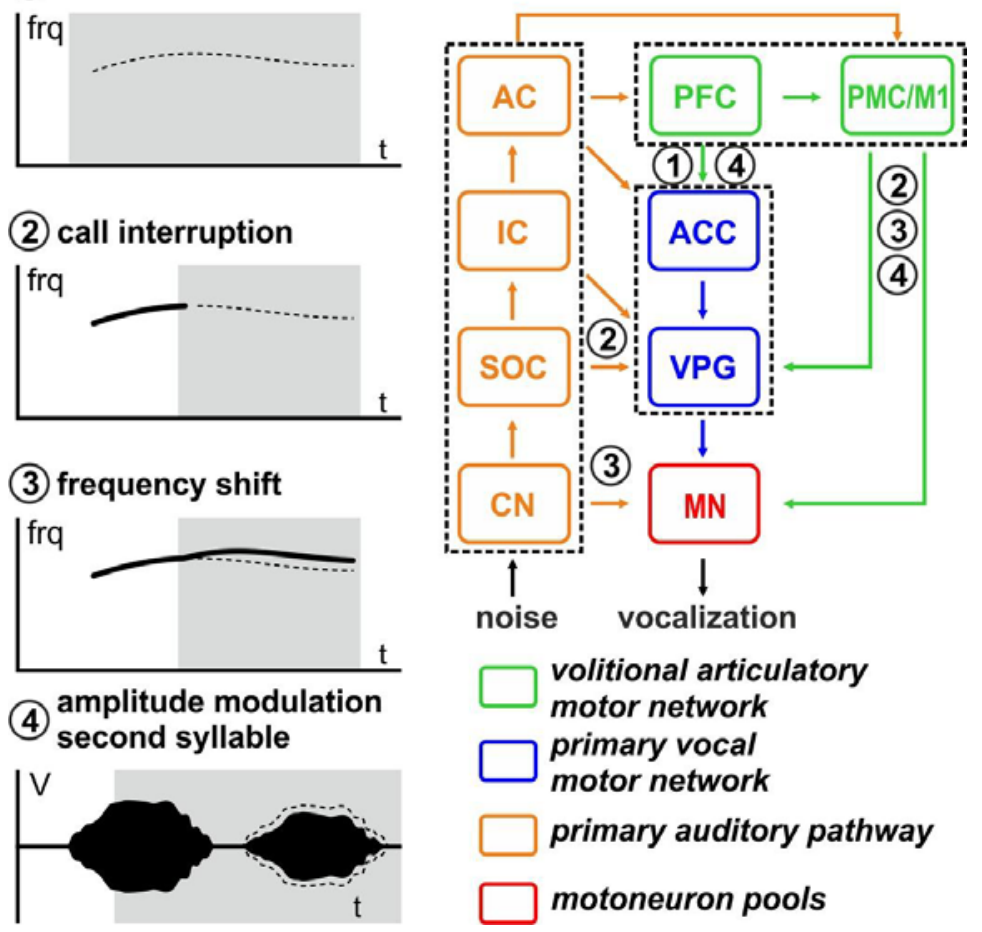

Figure 4: Hypothetical neuronal model for audio-vocal interaction. Call production might be affected by ambient noise at different brain levels. Audio-vocal integration mechanisms are known to happen between cortical and subcortical structures as well as via corticofugal projections. See text for further explanation. 


\section{Supplemental Figures}
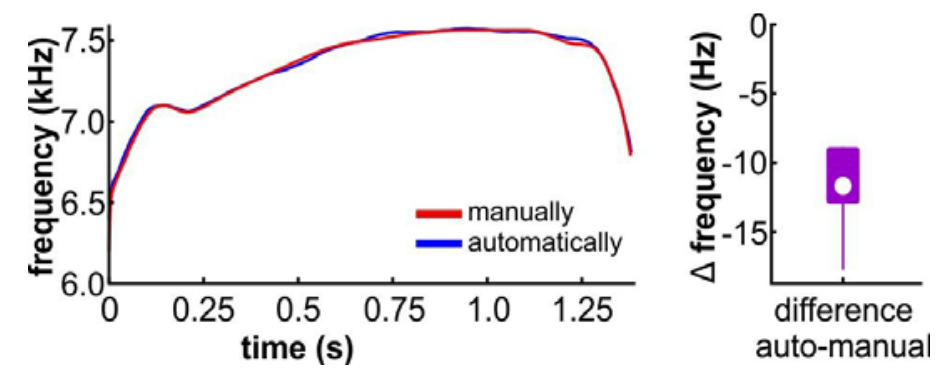

Figure S1: Comparison of automatically vs manually marked calls. (A) Frequency course $(\mathrm{Hz})$ over time (ms) of a manually (blue) and automatically (red) marked example phee call. (B) Mean $\Delta$ frequencies of automatically-manually marked calls. 

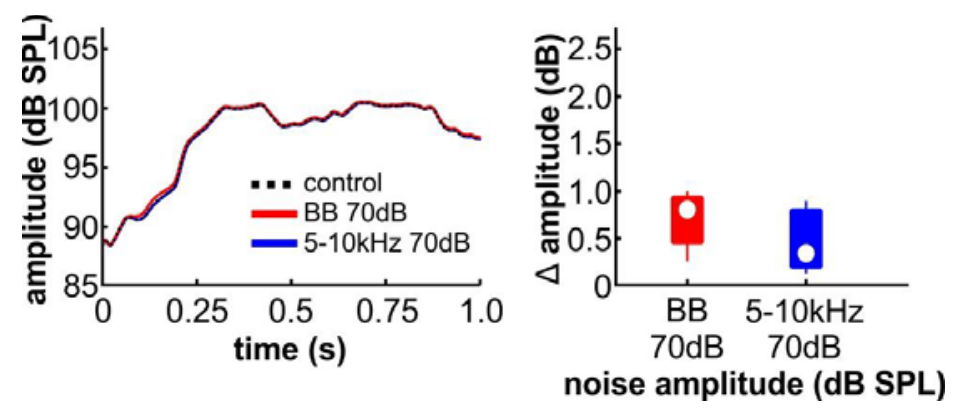

Figure S2: Test for noise subtraction accuracy. (A) Mean amplitude courses (dB SPL) for both $70 \mathrm{~dB}$ overlapping noise conditions $(5-10 \mathrm{kHz}$, blue; broadband, red) as well as for the control (no noise, black dashed) of 10 test phees over time (ms). (B) Maximum $\Delta$ amplitude (dB) compared to control calls of $70 \mathrm{~dB}$ broadband and $5-10 \mathrm{kHz}$ noise conditions. 

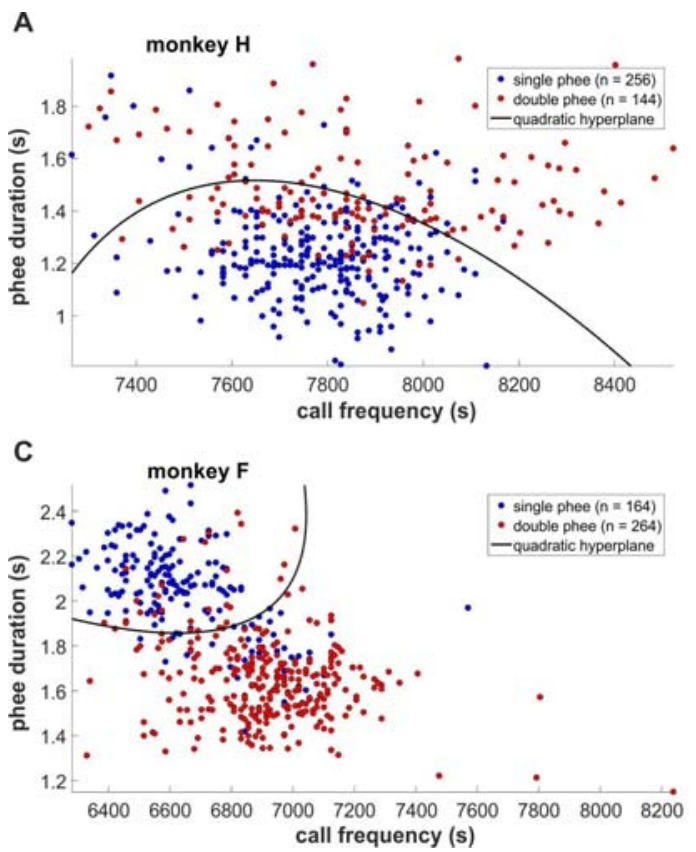

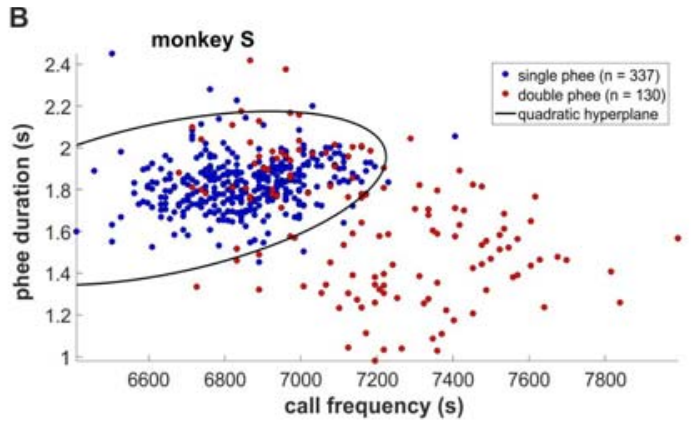

D

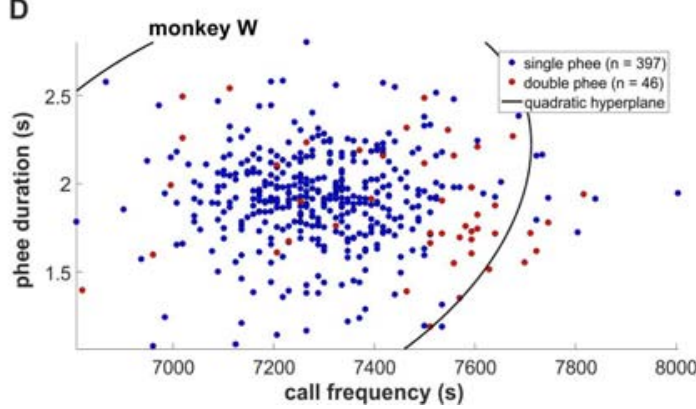

Figure S3: Single-double phee discrimination with a two-dimensional classifier. For single-double phee discrimination, first syllable fundamental peak frequencies $(\mathrm{Hz})$ at $25 \mathrm{~ms}$ after call onset and corresponding phee durations (s) were used. 
A
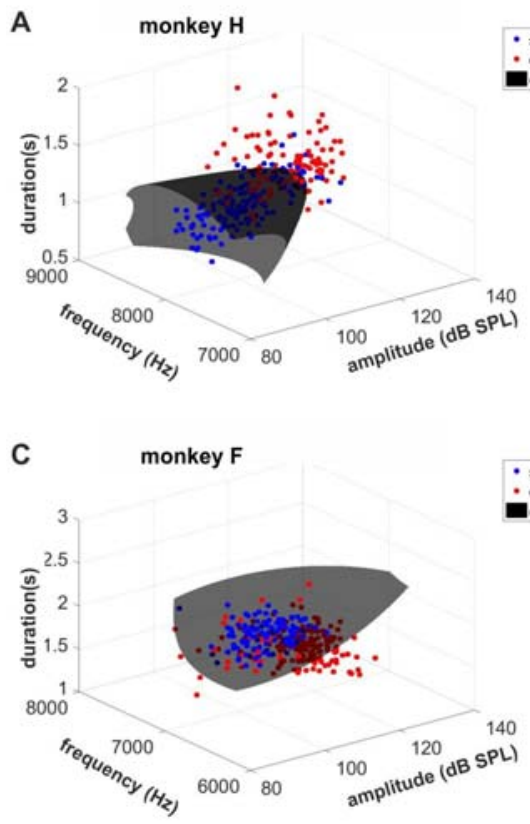
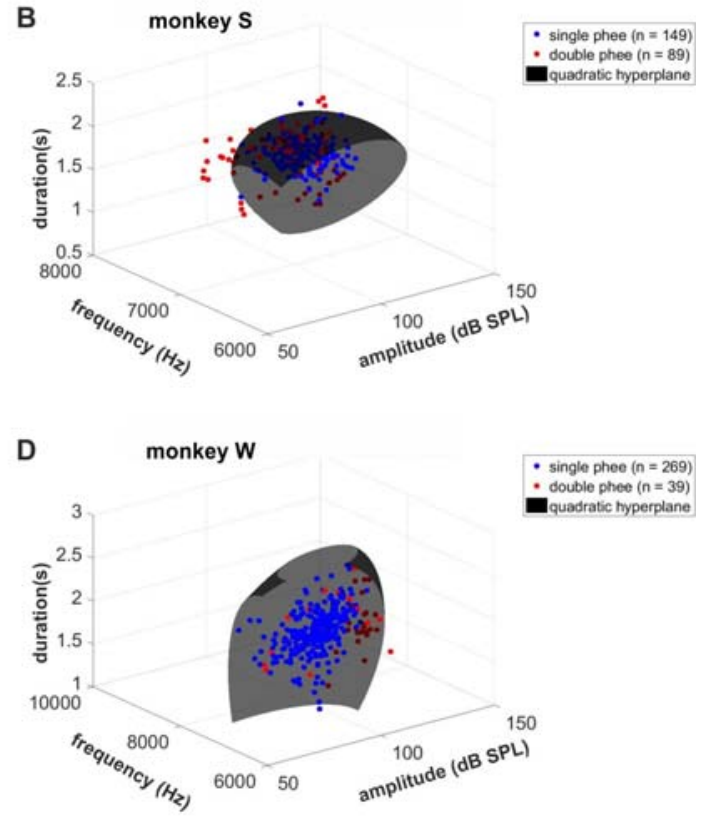

Figure S4: Single-double phee discrimination with a three-dimensional classifier. For single-double phee discrimination, first syllable fundamental peak frequencies $(\mathrm{Hz})$, as well as amplitudes (dB SPL) at $25 \mathrm{~ms}$ after call onset and corresponding phee durations (s), were used. 

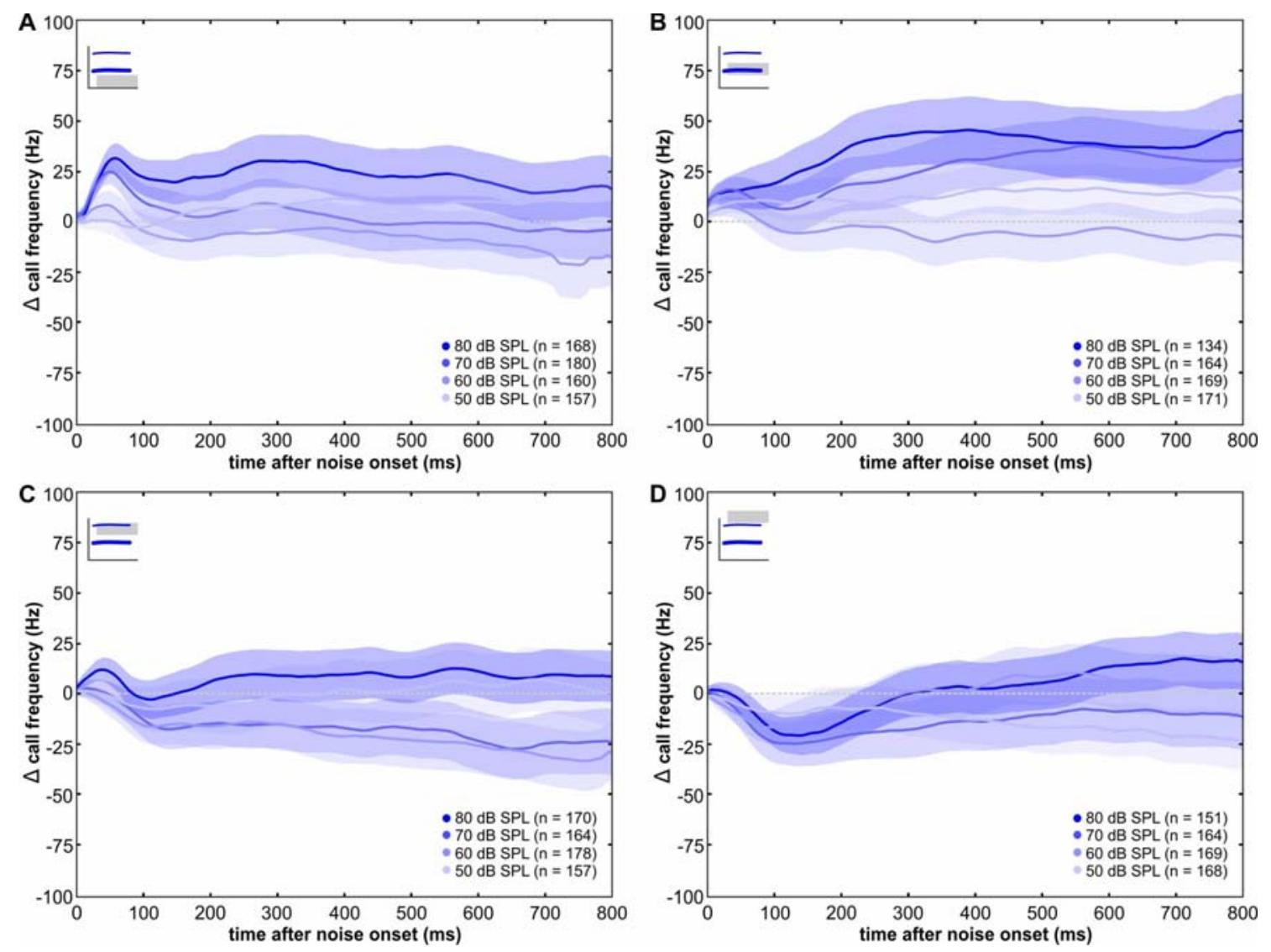

Figure S5: Frequency courses in response to noise bursts. First syllables mean $\Delta$ frequency courses $(\mathrm{Hz}) \pm \mathrm{SEM}$ of all amplitude conditions normalized to control data (dashed lines) during (A) $0.1-5.1 \mathrm{kHz},(\mathbf{B}) 5-10 \mathrm{kHz},(\mathbf{C}) 10-15 \mathrm{kHz}$, and (D) $16-21 \mathrm{kHz}$ noise conditions over time after noise onset (ms). 

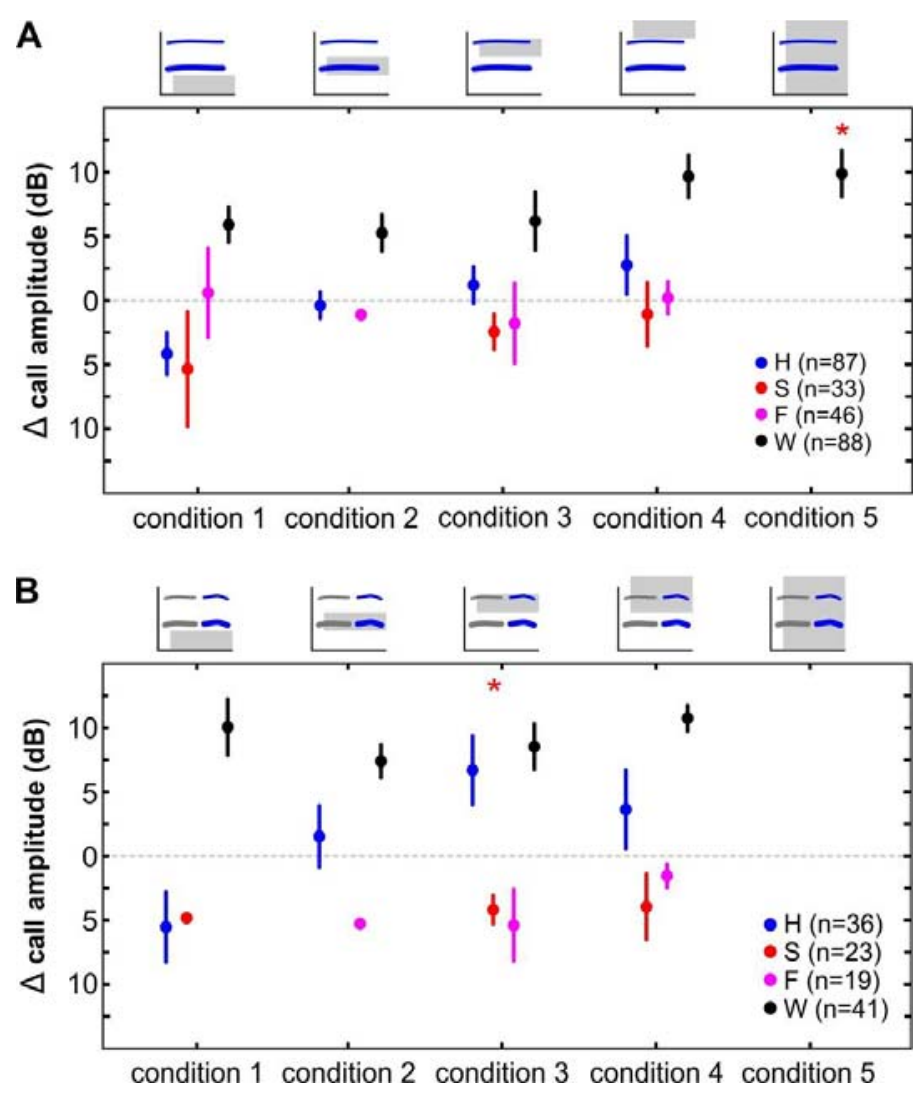

Figure S6: Amplitude shifts in response to noise bursts. Pooled maximum $\triangle$ amplitudes (dB) \pm SEM during $180 \mathrm{~s}$ noise per monkey per noise condition normalized to control data (dashed lines) (A) for first syllables and (B) for second syllables. Asterisks denote significant differences. 